Bull. Soc. math. France

129 (1), 2001, p. 91-113

\title{
PERTURBATION SINGULIÈRE \\ EN DIMENSION TROIS : CANARDS \\ EN UN POINT PSEUDO-SINGULIER NGUD
}

\author{
PAR ÉRIC BENOÎT
}

RÉsumÉ. - On étudie les systèmes différentiels singulièrement perturbés de dimension 3 du type

$$
\left\{\begin{array}{l}
\dot{x}=f(x, y, z, \varepsilon), \\
\dot{y}=g(x, y, z, \varepsilon), \\
\varepsilon \dot{z}=h(x, y, z, \varepsilon),
\end{array}\right.
$$

où $f, g, h$ sont analytiques quelconques. Les travaux antérieurs étudiaient les points réguliers où la surface lente $h=0$ est transverse au champ rapide vertical. C'est le domaine d'application du théorème de Tikhonov. Dans d'autres travaux antérieurs, on étudiait les singularités de certains types : plis et fronces de la surface lente, ainsi que certaines singularités plus compliquées, analogues aux points tournants en dimension inférieure : les points pseudo-singuliers cols. Génériquement, les seules singularités génériques non encore étudiées dans la littérature sont les points pseudo-singuliers nœuds.

Dans cet article, on étudie les points pseudo-singuliers nœuds où on montre l'existence d'une ou deux solutions surstables (c'est-à-dire assez régulières en $\varepsilon$ ). Quand le rapport de deux valeurs propres est entier, un phénomène nouveau et intéressant apparaît : la résonance.

Techniquement, on se ramène d'abord à une forme plus canonique, puis on montre l'existence de solutions formelles, en utilisant le théorème des fonctions implicites sur un opérateur entre espaces de Banach de séries Gevrey. Les séries obtenues sont alors Gevrey, et les théories de sommation de ces séries donnent les solutions surstables recherchées.

Texte reçu le 12 octobre 1999, accepté le 2 décembre 1999

ÉRIC BENOîT, Université de la Rochelle • E-mail : ebenoit@univ-lr.fr Url : http://www.univ-lr.fr/intranetULR.html

Classification mathématique par sujets (2000). - 34E15, 34M30, 34M60, 34E05, 34C20.

Mots clefs. - Canard, équation différentielle ordinaire, perturbation singulière, point tournant, série Gevrey, solution surstable. 
ABSTRACT (Singular perturbation, tridimensional case : canards on a pseudo-singular node point)

We study singularly perturbed system of differential equations like

$$
\left\{\begin{aligned}
& \dot{x}=f(x, y, z, \varepsilon), \\
& \dot{y}=g(x, y, z, \varepsilon), \\
& \varepsilon \dot{z}=h(x, y, z, \varepsilon),
\end{aligned}\right.
$$

where $f, g$ and $h$ are analytic functions. In known papers, regular points of the slow surface $h=0$ are studied. At this point, the fast flow (vertical) is tranverse to the slow surface. The Tikhonov's theorem can be applied here. In other papers, fold points and cusps of the slow surface were studied. The list of generic singularities contains also the pseudo-singular points which are connected to the turning points in lower dimension. They are (generically) saddle, focus or node. In the neighborhood of focus points, nothing happens, the saddle were studied in their papers, but the node points were never studied in the litterature.

In this paper, whe prove that generally, there exist two overstable (i.e. regular with respect $\varepsilon$ ) solutions. When the ratio between two eigenvalues is an integer, a resonance appears, and one of the two overstable solutions disappears.

Technically, we transform first the system into a more canonical equation. After that, we prove the existence of formal solutions, and, using the implicit function theorme on Banach spaces of Gevrey series, we can prove that the formal solution is Gevrey. The theory of summation of Gevrey series gives the over-stable solutions.

\section{Introduction}

La plupart des travaux sur les perturbations singulières d'équations différentielles ordinaires concernent les cas où la dimension de la variable lente est 1. Ce sont les équations du type

$$
\left\{\begin{aligned}
x^{\prime} & =f(x, z, \varepsilon), & & x \in \mathbb{R}, \\
\varepsilon z^{\prime} & =h(x, z, \varepsilon), & & z \in \mathbb{R}^{n} .
\end{aligned}\right.
$$

Les méthodes sont diverses : la géométrie avec les variétés invariantes (Dumortier, Roussarie, Jones [8]) ; l'analyse non standard (Benoît, Callot, Diener, Sari, etc., [2], [10]) ; le calcul asymptotique classique avec matching (Wasow, Vasil'eva, O'Malley,..., [14]) ; l'ajout à cela d'un brin d'analyse complexe (méthode de Kruskal-Segur); les propriétés Gevrey des solutions formelles (Malgrange, Ramis, Sibuya, Écalle,..., [12], [7]), etc. Toutes ces méthodes ont bien sûr des atouts différents, et leur utilisation simultanée permet d'avancer plus loin dans certains problèmes.

Dans le cas où la dimension de la variable lente est strictement supérieure à 1 ,

$$
\begin{cases}x^{\prime}=f(x, z, \varepsilon), & x \in \mathbb{R}^{p}, \\ \varepsilon z^{\prime}=h(x, z, \varepsilon), & z \in \mathbb{R}^{n},\end{cases}
$$

TOME $129-2001-\mathrm{N}^{\mathrm{O}} 1$ 
certains travaux se généralisent : le théorème de Tikhonov, les résultats de Vasili'eva, etc., mais ils font tous l'hypothèse que, le long des trajectoires lentes étudiées, le spectre de la partie linéaire du champ rapide évite zéro (ou même l'axe imaginaire). Ainsi, l'indice de stabilité des branches de variété lente ne change pas. Dans le cas contraire, les problèmes sont beaucoup plus délicats (généralisation des plis, et surtout des points tournants) et je ne connais pas de résultats généraux.

Dans [3], j'ai étudié certains problèmes apparaissant génériquement quand les dimensions lente et rapide sont respectivement 2 et 1 . J'avais utilisé des méthodes géométriques et l'analyse non standard. Ces méthodes n'avaient pas permis de résoudre le problème dans d'autres cas. Dans cet article, je me propose de compléter cette étude en étudiant les cas génériques non encore connus.

Le système de base est donc :

$$
\left\{\begin{array}{l}
x^{\prime}=f\left(x, y, z, \varepsilon^{2}\right), \\
y^{\prime}=g\left(x, y, z, \varepsilon^{2}\right) \\
\varepsilon^{2} z^{\prime}=h\left(x, y, z, \varepsilon^{2}\right) .
\end{array}\right.
$$

Dans l'écriture ci-dessus, il peut sembler étrange d'avoir mis $\varepsilon^{2}$ pour le petit paramètre. C'est pour simplifier la suite de la présentation.

Les méthodes géométriques non standard (voir [3], [4], [5]) ont donné sur ce type de systèmes un certain nombre de résultats généraux qui montraient l'importance des canards dans ces problèmes. Cependant, ils n'ont pas permis de répondre à une question fondamentale : l'existence de canards au voisinage d'un point pseudo-singulier de type nœud. Ce dernier point, résolu dans les pages qui suivent en utilisant des séries divergentes Gevrey a déjà été démontré simultanément et indépendamment par M. Wechselberger [15] à l'aide de méthodes géométriques utilisant des éclatements et des variétés centrales. Ce dernier, malgré l'utilisation de méthodes fondamentalement différentes, retrouve la nécessité des hypothèses de non résonance, et l'impossibilité de préciser le caractère global des canards.

1.1. Énoncé du problème et hypothèses. - Dans ce paragraphe, on reprend les définitions et notations de [3], [4], [5]. Dans ces anciens articles, le paramètre $\varepsilon$ était considéré comme fixé, infinitésimal (au sens de l'analyse non standard). Ici, comme chez $\mathrm{M}$. Wechselberger, $\varepsilon$ est une variable qui tend vers zéro. Chez M. Wechselberger, $\varepsilon$ reste réel positif, alors qu'ici, il sera tout d'abord formel, puis vivra dans des secteurs de $\mathbb{C}$ ayant l'origine pour sommet.

Les fonctions $f, g$ et $h$ sont supposées analytiques en $x, y$ et $z$ sur un domaine de $\mathbb{C}^{3}$. Pour simplifier les énoncés, on supposera que ce domaine est $\mathbb{C}^{3}$ tout entier. Encore pour simplifier, on supposera $f, g$ et $h$ analytiques par rapport à leur quatrième variable. En fait, il suffirait de supposer qu'elles sont analytiques pour $\varepsilon$ dans un certain secteur contenant l'axe réel positif et qu'elles ont un développement asymptotique Gevrey d'ordre approprié en $\varepsilon$.

BULLETIN DE LA SOCIÉtÉ MATHÉMATIQUE DE FRANCE 
La surface lente est la surface d'équation $h(x, y, z, 0)=0$. En un point non situé sur la surface lente, le champ de vecteurs donné par (1) est presque vertical (la composante verticale tend vers l'infini quand $\varepsilon$ tend vers zéro).

Les points génériques étudiés dans cet article sont les points où les hypothèses suivantes sont vérifiées ( $h_{z}$ désigne la dérivée partielle de $h$ par rapport à $z$, les autres notations sont analogues) :

$$
\begin{aligned}
& h=0, \quad h_{z}=0, \quad f h_{x}+g h_{y}=0, \\
& \mu \leq \lambda<0, \\
& \nabla h \neq 0, \quad h_{z z} \neq 0, \quad(f, g, h) \neq 0, \\
& \frac{\mu}{\lambda} \notin \mathbb{N} .
\end{aligned}
$$

Les égalités (2) caractérisent les points dits pseudo-singuliers. Les nombres $\lambda$ et $\mu$ seront définis précisément plus loin (voir le $\S 1.3$ ); ce sont les valeurs propres du linéarisé d'un champ de vecteurs en dimension 2 . Ils classifient les pseudo-singularités en cols, nœuds et foyers, les inégalités (3) caractérisent les points pseudo-singuliers nœuds. Les non-égalités (4) sont génériques et (5) est dite hypothèse de non-résonance. Elle est aussi générique et sera commentée dans la suite du travail.

En revenant à la problématique initiale des perturbations singulières, il est tout naturel de définir le système réduit par les équations :

$$
\left\{\begin{array}{l}
x^{\prime}=f(x, y, z, 0), \\
y^{\prime}=g(x, y, z, 0), \\
0=h(x, y, z, 0) .
\end{array}\right.
$$

C'est l'objet vers lequel on voudrait voir « converger » le système (1). Cependant, si on le définit comme une équation algébro-différentielle, les solutions vivent toutes sur la surface lente, et la « convergence» ne peut pas être définie correctement. On adopte donc la

DÉFINITION 1.1 (voir [3]). — On appelle candidat toute concaténation de segments verticaux, solutions de $\dot{z}=h(x, y, z, 0)$ et de solutions du champ de vecteurs défini sur la surface lente par le système réduit (6).

1.2. Résultats. - Il est facile de voir que la limite, quand $\varepsilon$ tend vers zéro, de n'importe quelle trajectoire du système (1) est un candidat. Ce théorème est énoncé proprement dans [3]. C'est une généralisation facile du théorème de Tikhonov qui combine sauts rapides, solutions lentes le long de la surface lente et passages sur le pli.

DÉfinition 1.2. - Parmi les candidats, on définit les candidats-canards : ils contiennent des bouts de solutions du système réduit, tracés sur la surface lente,

TOME $129-2001-\mathrm{N}^{\mathrm{O}} 1$ 
d'abord sur une nappe attractive (où $h_{z}<0$ ), puis sur une nappe répulsive (où $h_{z}>0$ ).

Dans sa définition d'un système contraint (voir [13]), Takens avait volontairement éliminé ces candidats car il semblait peu probable qu'ils soient limites de trajectoires de (1) (d'ailleurs cette affirmation est «numériquement » exacte : quand $\varepsilon$ est petit, il est très difficile de trouver numériquement des trajectoires voisines d'un candidat-canard). Si une famille $\gamma_{\varepsilon}$ de trajectoires de (1) converge (au sens $C^{0}$ ) vers un candidat-canard quand $\varepsilon$ tend vers zéro, on dit que $\gamma_{\varepsilon}$ est un canard.

$\mathrm{Au}$ voisinage d'un point pseudo-singulier nœud, il y a beaucoup de candidats canards (voir [5] et la figure ci-dessous). Nous allons montrer dans la suite de l'article que seuls quelques-uns sont limites de canards de (1).



FiguRE 1. Quelques candidats-canards

Plus précisément, dans cet article, le théorème principal est le théorème 1.5 ci-dessous, mais la difficulté est concentrée dans le théorème 1.4. Mais il nous faut d'abord rappeler la définition des séries Gevrey, adaptée à notre problème :

DÉFInItion 1.3. - Une solution formelle de (1) notée

$$
\hat{\gamma}(t)=\left\{\begin{array}{l}
\hat{x}(t)=\sum_{n \geq 0} x_{n}(t) \varepsilon^{n} \\
\hat{y}(t)=\sum_{n \geq 0} y_{n}(t) \varepsilon^{n} \\
\hat{z}(t)=\sum_{n \geq 0} z_{n}(t) \varepsilon^{n}
\end{array}\right.
$$

est dite Gevrey-2 si les fonctions $x_{n}, y_{n}$ et $z_{n}$ sont analytiques, au moins pour $|t|<r$, et si de plus, il existe des constantes $\alpha$ et $A$ telles que

$$
\left\|x_{n}\right\|_{n / 2},\left\|x_{n}^{\prime}\right\|_{n / 2},\left\|y_{n}\right\|_{n / 2},\left\|y_{n}^{\prime}\right\|_{n / 2},\left\|z_{n}\right\|_{n / 2},\left\|z_{n}^{\prime}\right\|_{n / 2}
$$

soient tous les six inférieurs à $A \alpha^{n} \Gamma\left(\frac{1}{2} n+1\right)$, où $\|\cdot\|_{n / 2}$ désigne une norme de type Nagumo sur les fonctions analytiques, définie précisément plus loin ( $§ .1$ ). 
THÉORÈme 1.4. - Soit $\left(x_{0}, y_{0}, z_{0}, 0\right)$ un point pseudo-singulier (2) nœud à canards (3) du système (1). Supposons que ce point est générique (4) et non résonnant (5). Alors, à translation temporelle près, il existe deux et seulement deux solutions formelles Gevrey de (1) notées $\hat{\gamma}_{\lambda}$ et $\hat{\gamma}_{\mu}$, vérifiant

$$
\left(\hat{\gamma}_{\lambda}\right)_{0}(0)=\left(\hat{\gamma}_{\mu}\right)_{0}(0)=\left(x_{0}, y_{0}, z_{0}\right)
$$

Le vecteur $\left(\hat{\gamma}_{\lambda}^{\prime}\right)_{0}(0)$ (resp. $\left.\left(\hat{\gamma}_{\mu}^{\prime}\right)_{0}(0)\right)$ est tangent au sous-espace propre du linéarisé de (7) correspondant à la valeur propre $\lambda$ (resp. $\mu)$.

THÉORÈme 1.5. - Soit $\left(x_{0}, y_{0}, z_{0}, 0\right)$ un point pseudo-singulier (2) noeud à canards (3) du système (1). Supposons que ce point est générique (4) et non résonnant (5). Alors, il existe en ce point deux canards notés $\gamma_{\lambda}$ et $\gamma_{\mu}$. Notons $\tilde{\gamma}_{\lambda}$ (resp. $\left.\tilde{\gamma}_{\mu}\right)$ la limite de $\gamma_{\lambda}\left(\right.$ resp. $\left.\gamma_{\mu}\right)$ quand $\varepsilon$ tend vers zéro. Le vecteur tangent $\grave{a} \tilde{\gamma}_{\lambda}$ (resp. $\tilde{\gamma}_{\mu}$ ) au point pseudo-singulier est le sous-espace propre $d u$ linéarisé de (7) correspondant à la valeur propre $\lambda$ (resp. $\mu$ ).

REMARQue 1.6. - En fait la convergence de $\gamma_{\lambda}$ vers $\tilde{\gamma}_{\lambda}$ est $C^{n}$ pour tout $n$. Il semble que tout canard de (1) qui converge au sens $C^{1}$ soit exponentiellement proche des deux trouvés dans le théorème. Cependant, il existe d'autres canards avec une convergence $C^{0}$ seulement : dans [5], la démonstration de ceci est faite dans un cas particulier; je conjecture que, moyennant quelques calculs fastidieux, cette démonstration s'étend au cas général évoqué ici.

1.3. Explication géométrique des hypothèses. - La surface lente est supposée régulière, c'est-à-dire que le gradient $\nabla h$ est différent de zéro en tous ses points. Cette hypothèse est générique au sens suivant : l'ensemble des points qui ne satisfont pas cette hypothèse est défini par quatre équations indépendantes quand on pose le problème comme cela est fait ici.

Le pli est l'ensemble des points de la surface lente où le plan tangent est vertical. Il a pour équation $h(x, y, z, 0)=h_{z}(x, y, z, 0)=0$. On suppose (génériquement) que c'est une courbe régulière, c'est-à-dire que, en tout point du pli, la matrice

$$
\left(\begin{array}{ccc}
h_{x} & h_{y} & h_{z} \\
h_{x z} & h_{y z} & h_{z z}
\end{array}\right)
$$

est de rang 2 .

Les fronces sont les points où la tangente au pli est verticale. Ils vérifient $h=h_{z}=h_{z z}=0$. Ils ne nous intéresseront pas dans cet article car dans le cas générique, le problème géométrique, presque trivial, est déjà résolu dans [3].

$\mathrm{Si}$, dans le système réduit (6), on dérive la troisième équation, puis on mul-

TOME $129-2001-\mathrm{N}^{\mathrm{O}} 1$ 
tiplie le champ par $-h_{z}$, on obtient la formulation suivante (où la dérivation se fait par rapport à une nouvelle variable temps) :

$$
\left\{\begin{array}{l}
\dot{x}=-f(x, y, z, 0) h_{z}(x, y, z, 0) \\
\dot{y}=-g(x, y, z, 0) h_{z}(x, y, z, 0) \\
\dot{z}=f(x, y, z, 0) h_{x}(x, y, z, 0)+g(x, y, z, 0) h_{y}(x, y, z, 0) .
\end{array}\right.
$$

La fonction $h$ est une intégrale première, et la dynamique ne nous intéresse que sur la surface lente. Les points stationnaires du système (7) sont de deux types. Dans le premier type, $f$ et $g$ s'annulent; ce sont alors des points stationnaires du système (6)), et génériquement ils n'appartiennent pas au pli. Ceux du second type sont les points du pli où $f h_{x}+g h_{y}$ s'annule. Ces derniers sont appelés pseudo-singularités du système (1). On étudie ensuite le type topologique de ces pseudo-singularités. Pour cela, on regarde le signe de la partie réelle des valeurs propres de la matrice jacobienne du système (7) (toutes les fonctions sont évaluées au point-pseudo-singulier, et $h_{z}$ est donc nul) :

$$
\left(\begin{array}{ccc}
-f h_{x z} & -f h_{y z} & -f h_{z z} \\
-g h_{x z} & -g h_{y z} & -g h_{z z} \\
f_{x} h_{x}+g_{x} h_{y}+f h_{x x}+g h_{x y} & g_{y} h_{y}+f_{y} h_{x}+g h_{y y}+f h_{y x} & f_{z} h_{x}+g_{z} h_{y}+f h_{x z}+g h_{y z}
\end{array}\right) .
$$

L'une des valeurs propres est nulle (conséquence de l'intégrale première $h$ ), les deux autres, notées $\lambda$ et $\mu$, caractérisent la nature du point pseudo-singulier : col, nœud ou foyer. Le cas des foyers ne pose aucune difficulté, celui des cols est étudié dans [3], [4], ainsi que celui des nœuds à faux-canards (c'est-à-dire $\lambda$ et $\mu$ strictement positifs). Dans cet article, on s'intéresse au seul cas restant : les nouds à canards, c'est-à-dire où $\lambda$ et $\mu$ sont tous deux réels négatifs.

1.4. Outils et plan de l'article. - Le cadre technique utilisé est la méthode de dilatation de Malgrange qu'on rappelle dans le paragraphe 3. Son application est faite dans les paragraphes 4 à 7 . Elle utilise un espace de Banach de séries formelles Gevrey. Les normes de type Nagumo utilisées interviennent dans des problèmes très voisins (voir [7])

Pour commencer, dans le paragraphe 2, le problème (1) est transformé en une équation non autonome du second ordre. Ceci permet une étude plus « analyse fonctionnelle complexe » du système, mais perd le caractère géométrique réel où le problème initial était posé.

Dans le paragraphe 8 , on applique le résultat formel du théorème 1.4 pour démontrer le théorème 1.5, par une méthode déjà mise au point dans [7].

Dans le paragraphe 9 , on fait quelques conjectures sur ces canards : sur leur domaine de définition et sur leur régularité.

BULletin DE LA SOCIÉtÉ MATHÉMATIQUE DE FRANCE 


\section{Transformations préliminaires}

2.1. Changements de variables réguliers en $\varepsilon$. - Les différents changements de variables de ce paragraphe sont réguliers en $\varepsilon$ (le terme utilisé en analyse non standard est : de classe $S^{0}$ ) : la géométrie du problème et le passage à la limite pour $\varepsilon$ tendant vers zéro ne sont pas modifiés. L'intérêt de ces transformations est de se placer dans un cas où les calculs sont moins lourds.

Quitte à faire une translation (indépendante de $\varepsilon$ ), on peut supposer que le point pseudo-singulier nœud est à l'origine.

Quitte à faire une rotation autour de l'axe $O z$, on peut supposer, en plus des hypothèses (2), que $h_{y}$ est nul à l'origine. On en déduit immédiatement qu'à l'origine, $f$ est nulle, alors que $g$ et $h_{x}$ ne le sont pas.

En multipliant $x, y$ et $z$ par des coefficients adéquats, on peut se ramener à $h_{x}=-1, g=1$ et $h_{z z}=-2$, toutes ses fonctions étant calculées à l'origine.

Un changement de variable du type $z \mapsto z+\alpha y$ permet de supposer que $h_{y z}=0$; un autre du type $x \mapsto x+\alpha y^{2}$ donne l'hypothèse $h_{y y}=0$; enfin un troisième, du type $x \mapsto x+\alpha \varepsilon^{2}$ donne $h_{\varepsilon^{2}}=0$

Le fait que la pseudo-singularité à l'origine soit du type nœud à canards se traduit par le fait que l'équation

$$
2 \lambda^{2}+f_{z} \lambda+f_{y}=0
$$

admet deux racines réelles négatives. Notons $\lambda$ celle qui a la plus petite valeur absolue. Le changement de variables et paramètres défini par

$$
(x, y, z, t, \varepsilon) \longmapsto\left(\frac{1}{\lambda^{2}} x, \frac{1}{\lambda^{2}} y, \frac{-1}{\lambda} z, \frac{1}{\lambda^{2}} t, \frac{1}{(-\lambda)^{3 / 2}} \varepsilon\right)
$$

permet finalement de se ramener au système ci-dessous, où $k$ est le rapport des deux valeurs propres. C'est un invariant du problème qui aura une importance considérable dans toute la suite. Par construction c'est un réel supérieur à 1 . Quand il est entier, une résonance se produit, et les résultats de cet article ne s'appliquent plus.

Proposition 2.1. - Le système initial (1) avec les hypothèses (2), (3) et (4) se ramène par des changements de variable polynomiaux d'inverse polynomiaux au système

$$
\left\{\begin{array}{l}
x^{\prime}=2 k y+2(k+1) z+F\left(x, y, z, \varepsilon^{2}\right), \\
y^{\prime}=1+G\left(x, y, z, \varepsilon^{2}\right), \\
\varepsilon^{2} z^{\prime}=-z^{2}-x+H\left(x, y, z, \varepsilon^{2}\right),
\end{array}\right.
$$

où le paramètre $k$ est réel, supérieur à 1 . La fonction $F$ (resp. $G, H)$ a une valuation pondérée supérieure ou égale à 2 (resp. 1,3). La pondération consiste $\grave{a}$ affecter $x$ du poids 2 , alors que $y, z$, et $\varepsilon$ ont pour poids 1 .

L'hypothèse de non résonance (5) se traduit par le fait que $k$ n'est pas entier.

TOME $129-2001-\mathrm{N}^{\mathrm{O}} 1$ 
2.2. Réduction à une équation du second ordre. - Pour simplifier encore le système précédent, on fait une dernière transformation permettant de supposer que $G=0$. Il s'agit de diviser le temps par $1+G$. Ceci n'est possible que dans un domaine où la fonction $G$ reste différente de -1 . On est donc obligé de se restreindre à un certain voisinage de l'origine $(e n(x, y, z, \varepsilon))$; on perd donc ici tout espoir de chasser directement les plus grands canards (c'est-à-dire les trajectoires qui convergeraient vers des segments maximaux de solutions du système réduit). D'un point de vue pratique, cela permet de remplacer $y$ par $t$, et d'obtenir un système non autonome de deux équations.

Dans la troisième équation du système (8), la dérivée à l'origine par rapport à $x$ est -1 . On peut donc, dans un certain voisinage, exprimer $x$ grâce au théorème des fonctions implicites, en fonction des autres variables. On définit ainsi la fonction $\xi$ par

$$
x=-z^{2}-\varepsilon^{2} z^{\prime}+\xi\left(y, z, \varepsilon^{2}, \varepsilon^{2} z^{\prime}\right)
$$

où la valuation pondérée de $\xi$ est au moins 3 (le poids de $z^{\prime}$ est nul).

En dérivant cette dernière égalité et en remplaçant $x^{\prime}$ avec la première équation de $(8)$, on a

$$
-2 z z^{\prime}-\varepsilon^{2} z^{\prime \prime}+\xi_{y}+\xi_{z} z^{\prime}+\xi_{\varepsilon^{2} z^{\prime}} \varepsilon^{2} z^{\prime \prime}=2 k y+2(k+1) z+F\left(\xi, y, z, \varepsilon^{2}\right) .
$$

Remarquons que le poids de $\xi_{y}$ et celui de $\xi_{z}$ est 2 , alors que celui de $\xi_{\varepsilon^{2} z^{\prime}}$ est 1 . En divisant alors par $1-\xi_{\varepsilon^{2} z^{\prime}}$ qui est non nul dans un voisinage de l'origine, on obtient la proposition suivante :

Proposition 2.2. - Le système initial (1) avec les hypothèses (2), (3) et (4) est équivalent, dans un voisinage de $(x, y, z, \varepsilon)=0$ à une équation non autonome du second ordre:

(9) $\frac{1}{2} \varepsilon^{2} z^{\prime \prime}+z z^{\prime}+k t+(k+1) z=\Phi_{0}\left(t, \varepsilon, z, \varepsilon^{2} z^{\prime}\right)+z^{\prime} \Phi_{1}\left(t, \varepsilon, z, \varepsilon^{2} z^{\prime}\right)$

De plus, les deux fonctions $\Phi_{0}$ et $\Phi_{1}$ sont de valuation pondérée au moins égale à 2. (Rappelons que le poids de $z^{\prime}$ est nul, alors que celui de $t$, $\varepsilon$ et $z$ est 1.)

\section{Preuve du théorème $\mathbf{1 . 4}$}

Démonstration. - Elle se fait par la méthode de dilatation. L'idée de cette méthode est due à B. Malgrange [11] ; elle m'a été suggérée par C. Zhang.

Soient $\mathcal{B}_{2}$ et $\mathcal{B}_{1}$ deux espaces de Banach. Les éléments de $\mathcal{B}_{2}$ et de $\mathcal{B}_{1}$ sont des fonctions ou des séries formelles de deux variables $t$ et $\varepsilon$. Les opérations de multiplication et de dérivation par rapport à $t$ sont donc bien définies. Soit $A$ l'opérateur défini par :

$A(\delta, z)=\frac{\delta^{2} \varepsilon^{2}}{2} z^{\prime \prime}+z z^{\prime}+k t+(k+1) z-\Phi_{0}\left(t, \delta \varepsilon, z, \delta^{2} \varepsilon^{2} z^{\prime}\right)-z^{\prime} \Phi_{1}\left(t, \delta \varepsilon, z, \delta^{2} \varepsilon^{2} z^{\prime}\right)$ 
On suppose que $A$, défini sur $\mathbb{C} \times \mathcal{B}_{2}$, prend ses valeurs dans $\mathcal{B}_{1}$. L'intérêt de cet opérateur réside dans la remarque évidente suivante :

Proposition 3.1. - Si $\delta \neq 0$ et si $A(\delta, z)=0$, alors la fonction (ou série formelle) qui à $(t, \varepsilon)$ associe $z(t, \varepsilon / \delta)$ est solution de l'équation (9).

Le principe de la méthode est alors le suivant :

1) Résoudre l'équation $A(0, z)=0$. On note $z_{0}$ une solution. Ce travail sera fait dans le paragraphe 4 .

2) Montrer que $A$ est continûment différentiable au voisinage du point $\left(0, z_{0}\right)$. Ce travail sera fait dans le paragraphe 6 .

3) Calculer la différentielle partielle de $A$ par rapport à $z$ au point $\left(0, z_{0}\right)$. Elle sera notée $L$. Ce travail sera aussi fait dans le paragraphe 6 .

4) Vérifier que $L$ est inversible. Ce travail sera fait dans le paragraphe 7.

5) Appliquer le théorème des fonctions implicites pour trouver une fonction $\zeta$ de $\mathbb{C}$ dans $\mathcal{B}_{2}$ définie au voisinage de zéro, et telle que $A(\delta, \zeta(\delta))$ soit identiquement nulle.

6) On en déduit l'existence d'un élément $\zeta(\delta)$ (une seule valeur de $\delta$ suffit) satisfaisant les hypothèses de la proposition 3.1 .

7) En appliquant la proposition 3.1 , on construit un élément de $\mathcal{B}_{2}$, solution de l'équation (9).

On aura alors montré l'existence d'un nombre $\delta$ non nul et d'une série formelle $z=\sum z_{n}(t) \varepsilon^{n}$ élément de $\mathcal{B}_{2}$ tels que $z(t, \varepsilon / \delta)$ soit solution de (9). Ainsi, la série formelle

$$
\hat{z}=\sum\left(\frac{z_{n}(t)}{\delta^{n}}\right) \varepsilon^{n}
$$

est solution de (9). L'appartenance à $\mathcal{B}_{2}$ de cette série formelle impliquera les majorations Gevrey de la définition 1.3, avec $\alpha=1 / \delta$.

En reprenant à l'envers les transformations du paragraphe 2, on obtient une solution formelle Gevrey de (1), tangente au sous-espace propre associé à la valeur propre $\lambda$.

Pour déterminer l'autre solution, tangente au sous-espace propre associé à la valeur propre $\mu$, on utilise exactement les mêmes arguments. Au préalable, on échange les notations $\lambda$ et $\mu$, ce qui transforme $k$ en $1 / k$. Remarquons que $1 / k$ n'est jamais entier, et que l'hypothèse de non-résonance est donc inutile pour étudier cette deuxième solution.

Ceci termine la preuve du théorème 1.4 énoncé dans l'introduction.

TOME $129-2001-\mathrm{N}^{\mathrm{O}} 1$ 


\section{Approximation à l'ordre 0}

Il s'agit de résoudre l'équation $A(0, z)=0$. On peut aussi interpréter ce même problème comme la recherche de l'approximation à l'ordre 0 d'une trajectoire de (8), analytique en $t$. L'équation à résoudre s'écrit :

$$
z z^{\prime}+k t+(k+1) z-\varphi_{0}(t, z)-z^{\prime} \varphi_{1}(t, z)=0
$$

où

$$
\varphi_{0}(t, z)=\Phi_{0}(t, 0, z, 0) \quad \text { et } \varphi_{1}(t, z)=\Phi_{1}(t, 0, z, 0)
$$

sont de valuation au moins 2 à l'origine.

Proposition 4.1. - Soit $k$ un réel non entier supérieur à 1 . Soit $\varphi_{0}(t, z)$ et $\varphi_{1}(t, z)$ deux fonctions analytiques de valuation au moins 2 à l'origine. L'équation (10), avec la condition initiale $z(0)=0$ admet exactement deux solutions analytiques pour $t$ dans un voisinage de zéro. La dérivée à l'origine vaut -1 pour l'une, et $-k$ pour l'autre.

Dans toute la suite, nous noterons $z_{0}$ la solution dont la dérivée est -1 .

Démonstration. — Dans le plan $(t, z)$, l'équation (10) s'écrit :

$$
\left\{\begin{array}{l}
\left(-z+\varphi_{1}(t, z)\right) \frac{\mathrm{d} t}{\mathrm{~d} t}=-z+\varphi_{1}(t, z), \\
\left(-z+\varphi_{1}(t, z)\right) \frac{\mathrm{d} z}{\mathrm{~d} t}=k t+(k+1) z-\varphi_{0}(t, z) .
\end{array}\right.
$$

En changeant de temps, on obtient le système

$$
\left\{\begin{array}{l}
\frac{\mathrm{d} t}{\mathrm{~d} \tau}=-z+\varphi_{1}(t, z) \\
\frac{\mathrm{d} z}{\mathrm{~d} \tau}=k t+(k+1) z-\varphi_{0}(t, z) .
\end{array}\right.
$$

Bien sûr, l'origine du système (11) est un point stationnaire, mais on s'intéressera aux courbes analytiques invariantes passant par l'origine. En effet, une telle courbe invariante (si la tangente à l'origine n'est pas verticale) est le graphe d'une fonction $z(t)$ analytique, solution de l'équation (10).

Pour étudier le système (11) au voisinage de l'origine, on regarde la partie linéaire. Celle-ci ne dépend pas des fonctions $\varphi_{0}$ et $\varphi_{1}$. Ses valeurs propres sont 1 et $k$. Comme $k$ est non entier supérieur à 1 , les valeurs propres appartiennent au domaine de Poincaré et il n'y a pas de résonance. Le théorème de Poincaré (voir [1]) s'applique : le système est localement holomorphiquement conjugué à sa partie linéaire. Or celle-ci possède exactement deux courbes invariantes analytiques passant par l'origine : les deux sous-espaces propres. La tangente à l'origine est donnée par les vecteurs propres $(1,-1)$ et $(1,-k)$. 
REMARQUE 4.2. - Si $k$ est entier positif, les valeurs propres appartiennent encore au domaine de Poincaré, mais il y a résonance. Il reste alors une unique solution analytique, ayant $-k$ pour dérivée à l'origine, mais l'autre solution, génériquement, n'existe pas.

\section{Espaces de Banach appropriés}

Tout ce paragraphe est dévolu à la construction et à l'étude des espaces de Banach $\mathcal{B}_{1}$ et $\mathcal{B}_{2}$. Leurs éléments sont des séries formelles en puissance de $\varepsilon$ dont les coefficients sont des fonctions analytiques de $t$ ayant un rayon de convergence au moins égal à $r$. Je remercie R. Schäfke pour les idées qu'il m'a suggérées.

5.1. Notations. - On utilise comme outil de base les mêmes normes de Nagumo modifiées que dans [7].

Soient $0<\rho<r$ des paramètres fixés une fois pour toutes. On suppose que $r$ est inférieur au rayon de convergence de la fonction $z_{0}$ trouvée au $\S 4$. Ainsi, $z_{0}$ appartiendra à l'espace de Banach $\mathcal{B}_{2}$ défini ci-dessous.

Soit $f=\sum_{n \in \mathbb{N}} f_{n}(t) \varepsilon^{n}$ une série formelle en puissances de $\varepsilon$ dont les coefficients $f_{n}$ sont des fonctions analytiques ayant un rayon de convergence supérieur ou égal à $r$. On note

$$
d(t)=\left\{\begin{array}{lll}
r-|t| & \text { si } & \rho \leq|t| \leq r \\
r-\rho & \text { si } & 0 \leq|t| \leq \rho .
\end{array}\right.
$$

Pour $m$ positif ou nul, non nécessairement entier, et pour $g$ une fonction analytique de rayon de convergence supérieur ou égal à $r$, on note

$$
\|g\|_{m}=\sup _{0 \leq|t|<r}|g(t)| \cdot d(t)^{m}
$$

$\|\cdot\|_{m}$ est une norme de Nagumo modifiée, comme dans [7]. Enfin, on note

$$
\|f\|_{1}=6 \sup _{n \in \mathbb{N}} \frac{\left\|f_{n}\right\|_{n / 2}}{\Gamma\left(\frac{1}{2} n+1\right)}, \quad\|f\|_{2}=2 \max \left(\|f\|_{1},\left\|f_{t}\right\|_{1}\right) .
$$

L'espace $\mathcal{B}_{1}$ est l'ensemble des $f$ tels que $\|f\|_{1}$ soit borné. De même $\mathcal{B}_{2}$ est l'ensemble des $f$ tels que $\|f\|_{2}$ soit borné. Ce sont des espaces de Banach.

\subsection{Lemmes de majoration}

LEMme 5.1. - Les normes $\|\cdot \mid\|_{1}$ et $\|\cdot\|_{2}$ sont des normes d'algèbre.

Démonstration. - Soient $f$ et $g$ dans $\mathcal{B}_{1}$, soit $h=f g$. Une majoration donne

$$
\begin{aligned}
\left\|h_{n}\right\|_{n / 2} & =\left\|\sum_{p+q=n} f_{p} g_{q}\right\|_{n / 2} \leq \sum_{p+q=n}\left\|f_{p}\right\|_{p / 2} \cdot\left\|g_{q}\right\|_{q / 2} \\
& \leq \frac{1}{36}\|f\|_{1} \cdot\|g\|_{1} \sum_{p+q=n} \Gamma\left(\frac{p}{2}+1\right) \Gamma\left(\frac{q}{2}+1\right) .
\end{aligned}
$$

TOME $129-2001-\mathrm{N}^{\mathrm{O}} 1$ 
Une majoration combinatoire (les termes extrêmes de la somme sont les termes dominants) donne

$$
\sum_{p+q=n} \Gamma\left(\frac{p}{2}+1\right) \Gamma\left(\frac{q}{2}+1\right)<c \Gamma\left(\frac{n}{2}+1\right)
$$

pour tout $n$, où $c$ est une constante qu'on peut facilement majorer (par exemple par 6). On en déduit facilement que $\|h\|_{1} \leq\|f\|_{1} \cdot\|g\|_{1}$.

Pour l'espace $\mathcal{B}_{2}$, ce n'est qu'un corollaire facile :

$$
\begin{aligned}
\left\|\frac{\mathrm{d}}{\mathrm{d} t}(f g)\right\| \|_{1} & \leq\left\|f^{\prime} g\right\|_{1}+\left\|f g^{\prime}\right\|_{1} \leq\left\|f^{\prime}\right\|_{1} \cdot\|g\|_{1}+\|f \mid\|_{1} \cdot\left\|g^{\prime}\right\|_{1} \\
& \leq \frac{1}{2}\|f\|_{2} \cdot\|g\|_{2} .
\end{aligned}
$$

On peut remarquer que les coefficients 2 et 6 mis dans la définition des normes ont été choisis pour éviter des coefficients dans l'énoncé de ce lemme. Ils peuvent être « améliorés », mais ceci ne présente sans doute pas grand intérêt.

LEMME 5.2. - Soit $\Phi\left(z_{1}, \ldots, z_{n}\right)$ une fonction analytique sur un polydisque de rayon $R=\left(r_{1}, \ldots, r_{n}\right)$. Soit $f_{i}$ des éléments de $\mathcal{B}_{1}$. On suppose que $\left\|f_{i}\right\|_{1}<r_{i}$. La série formelle $\Phi\left(f_{1}, \ldots, f_{n}\right)$ est alors un élément de $\mathcal{B}_{1}$.

Démonstration. - Donnons d'abord quelques notations : si $i=\left(i_{1}, \ldots, i_{n}\right)$ est un multi-indice, on note $|i|=i_{1}+\cdots+i_{n}$. Si $z$ est un $n$-uplet d'éléments de $\mathbb{C}$, on note $z^{i}=z_{1}^{i_{1}} \cdots z_{n}^{i_{n}}$. Le développement en série de $\Phi$ s'écrit :

$$
\Phi(z)=\sum_{k=0}^{\infty} \Phi_{k}(z) \quad \text { où } \quad \Phi_{k}(z)=\sum_{|i|=k} a_{i} z^{i} .
$$

Notons $\Phi^{+}$la fonction analytique sur le même polydisque, définie par

$$
\Phi^{+}(z)=\sum_{k=0}^{\infty} \Phi_{k}^{+}(z) \quad \text { où } \quad \Phi_{k}^{+}(z)=\sum_{|i|=k}\left|a_{i}\right| z^{i} .
$$

Comme $\Phi_{k}$ est un polynôme, on sait que $\Phi_{k}\left(f_{1}, \ldots, f_{n}\right)$ appartient à $\mathcal{B}_{1}$ et que

$$
\left\|\mid \Phi_{k}\left(f_{1}, \ldots, f_{n}\right)\right\|_{1} \leq \Phi_{k}^{+}\left(\left\|f_{1}\right\|_{1}, \ldots,\left\|f_{n}\right\|_{1}\right) .
$$

La série $\sum_{k} \Phi_{k}(f)$ est donc normalement convergente. Comme $\mathcal{B}_{1}$ est complet, elle converge dans $\mathcal{B}_{1}$, et on a

$$
\left\|\Phi\left(f_{1}, \ldots, f_{n}\right)\right\|_{1} \leq \Phi^{+}\left(\left\|f_{1}\right\|_{1}, \ldots,\left\|f_{n}\right\|_{1}\right) .
$$

LEMME 5.3. - Soit $\Phi$ une fonction analytique d'une variable $z$ sur un disque de rayon $R$. La fonction qui à $f$ associe $\Phi(f)$ est définie sur la boule de rayon $R$ de $\mathcal{B}_{1}$, à valeurs dans $\mathcal{B}_{1}$. Elle est continûment différentiable et sa différentielle en $f$ est la multiplication par $\Phi^{\prime}(f)$. 
Démonstration. — On définit la fonction analytique

$$
\Delta(z, u)=\frac{\Phi(z+u)-\Phi(z)}{u} \text { et } \Delta(z, 0)=\Phi^{\prime}(z) .
$$

C'est une fonction analytique pour $|z|<R,|u|<R-|z|$. On substitue à $z$ et $u$ des séries formelles $f$ et $h$, éléments de $\mathcal{B}_{1}$, de normes convenables. On a alors

$$
\Phi(f+h)-\Phi(f)=h \Delta(f, h) .
$$

Le lemme 5.2 donne alors la majoration

$$
\|\mid \Phi(f+h)-\Phi(f)\|\left\|_{1}<\Delta^{+}\left(\|f\|_{1},\|h\|_{1}\right) \cdot\right\| h \|_{1} .
$$

$\Phi$ est donc continue de $\mathcal{B}_{1}$ vers $\mathcal{B}_{1}$.

Les mêmes arguments, appliqués à la fonction $\Phi(f+h)-\Phi(f)-\Phi^{\prime}(f) h$ montrent que cette dernière a une norme en $\|h\|_{1}^{2}$, et donc prouvent la différentiabilité de $\Phi$. L'écriture même de la différentielle montre sa continuité.

LEMme 5.4. - Soit $f$ un élément de $\mathcal{B}_{1}$. On a alors

$$
\left\|\varepsilon^{2} f^{\prime}\right\|_{1} \leq \frac{e}{\sqrt{2}}\|f\|_{1} .
$$

C'est pour avoir ce résultat qu'ont été introduites des normes de type Nagumo. Un lemme très voisin a été utilisé dans les travaux de [7].

Démonstration. — C'est une conséquence immédiate d'un lemme de Malgrange. Ce dernier permet de majorer la fonction analytique $f^{\prime}$ avec la fonction $f$. On utilise pour cela la formule de Cauchy donnant la dérivée. On majore, mais le prix à payer pour ne pas restreindre le disque de validité des majorations est l'introduction de la fonction $d(t)$, donc des normes de Nagumo. On obtient alors

$$
\left\|f_{n-2}^{\prime}\right\|_{n / 2} \leq \frac{n}{2} e\left\|f_{n-2}\right\|_{n / 2-1}
$$

dont on déduit facilement le résultat du lemme.

DÉfinition 5.5. - Soit $f$ un élément de $\mathcal{B}_{1}$. On définit les opérateurs $S$ et $Z$ par :

$$
(Z f)(t, \varepsilon)=f(0, \varepsilon), \quad(S f)(t, \varepsilon)=\frac{f(t, \varepsilon)-f(0, \varepsilon)}{t} .
$$

Lemme 5.6. - L'opérateur $S$ va de $\mathcal{B}_{1}$ vers $\mathcal{B}_{1}$. De plus, il est borné :

$$
\|S f\|_{1} \leq \frac{2}{\rho}\|f\|_{1} .
$$

Démonstration. — Elle est donnée dans [7]. C'est pour avoir cette majoration que les normes de Nagumo ont été modifiées. (Pour la norme de Nagumo originale, on avait $d(t)=r-|t|$.)

TOME $129-2001-\mathrm{N}^{\mathrm{O}} 1$ 
Lemme 5.7. - L'opérateur $Z$ va de $\mathcal{B}_{1}$ vers $\mathcal{B}_{1}$. De plus, il est borné :

$$
\|Z f\|_{1} \leq\|f\|_{1}
$$

Démonstration. - Se démontre par application directe des définitions.

LEMme 5.8. - Soient $f$ un élément de $\mathcal{B}_{1}$ et $p$ un entier. On a :

$$
f=\sum_{i=0}^{p} t^{i} Z S^{i} f+t^{p+1} S^{p+1} f .
$$

Démonstration. — C'est une écriture de la formule de Taylor. Elle se démontre par récurrence à partir de $f=Z f+t S f$.

\section{Différentiabilité de l'opérateur $A$}

En fait, la quasi-totalité du travail a déjà été faite dans les lemmes du paragraphe précédent (en particulier les lemmes 5.2 et 5.3 ).

En effet, l'application de $\mathcal{B}_{1}$ dans $\mathcal{B}_{1}$ qui à $z$ associe $\varepsilon^{2} z^{\prime}$ est linéaire bornée (voir lemme 5.4), elle est donc de classe $C^{1}$. L'application identité de $\mathcal{B}_{2}$ dans $\mathcal{B}_{1}$ est linéaire bornée, elle est aussi de classe $C^{1}$. Par composition et application du lemme 5.3, la fonction de $\mathcal{B}_{2}$ dans $\mathcal{B}_{1}$ qui à $z$ associe $\Phi_{0}\left(t, \delta \varepsilon, z, \delta^{2} \varepsilon^{2} z^{\prime}\right)$ est de classe $C^{1}$, et quand $\delta=0$, sa différentielle en $z_{0}$ est donnée par

$$
u \longmapsto \frac{\partial \Phi_{0}}{\partial z}\left(t, 0, z_{0}, 0\right) u \text {. }
$$

L'application définie de $\mathbb{C} \times \mathcal{B}_{2}$ dans $\mathcal{B}_{1}$ qui à $(\delta, z)$ associe $\Phi_{0}\left(t, \delta \varepsilon, z, \delta^{2} \varepsilon^{2} z^{\prime}\right)$ a des différentielles partielles continues. Elle est donc de classe $C^{1}$.

L'application de $\mathcal{B}_{2}$ dans $\mathcal{B}_{1}$ qui à $z$ associe $z^{\prime}$ est linéaire bornée, elle est donc de classe $C^{1}$.

Pour finir, l'application qui à $z$ associe $\varepsilon^{2} z^{\prime \prime}$ est linéaire bornée en tant qu'application de $\mathcal{B}_{2}$ dans $\mathcal{B}_{1}$. Par multiplication et addition de fonctions $C^{1}$, l'opérateur $A$ est $C^{1}$.

Sa différentielle partielle par rapport à $z$ au point $\left(0, z_{0}\right)$ est donnée par

$$
\begin{aligned}
L(u)=u z_{0}^{\prime}+z_{0} u^{\prime}+(k+1) u-\frac{\partial \Phi_{0}}{\partial z}\left(t, 0, z_{0}, 0\right) u \\
\quad-\Phi_{1}\left(t, 0, z_{0}, 0\right) u^{\prime}-z_{0}^{\prime} \frac{\partial \Phi_{1}}{\partial z}\left(t, 0, z_{0}, 0\right) u \\
=\left(z_{0}-\Phi_{1}\left(t, 0, z_{0}, 0\right)\right) u^{\prime} \\
\quad+\left(z_{0}^{\prime}+k+1-\frac{\partial \Phi_{0}}{\partial z}\left(t, 0, z_{0}, 0\right)-z_{0}^{\prime} \frac{\partial \Phi_{1}}{\partial z}\left(t, 0, z_{0}, 0\right)\right) u .
\end{aligned}
$$

On écrira en résumé

$$
L(u)=-t \varphi u^{\prime}+k \psi u
$$

où les fonctions $\varphi$ et $\psi$ sont définies par la formule précédente. On remarque que ce sont des fonctions analytiques d'une seule variable $t$, indépendantes 
de $\varepsilon$. Comme $\Phi_{0}$ et $\Phi_{1}$ sont de valuation au moins 2 , et comme $z_{0}(0)$ est nul, on remarque aussi que la mise en facteur de $t$ dans le coefficient de $u^{\prime}$ était licite, que $\varphi(0)=-z_{0}^{\prime}(0)$ et $\psi(0)=\left(z_{0}^{\prime}(0)+k+1\right) / k$.

Ainsi, puisque $z_{0}^{\prime}(0)=-1$, on a $\varphi(0)=1$ et $\psi(0)=1$.

\section{7. Étude de l'opérateur linéaire}

C'est dans ce paragraphe qu'apparaît la nécessité du choix des normes sur $\mathcal{B}_{1}$ et $\mathcal{B}_{2}$. C'est le cœur de la preuve du théorème d'existence de canards formels Gevrey dans l'équation (1). La proposition ci-dessous doit être appliquée à l'opérateur trouvé dans le paragraphe 6 , mais elle est plus générale, car on ne retient qu'un nombre très restreint de propriétés des fonctions $\varphi$ et $\psi$.

Proposition 7.1. - Soit $\varphi$ et $\psi$ deux éléments de $\mathcal{B}_{1}$. On suppose que $1 / \varphi$ appartient aussi à $\mathcal{B}_{1}$, que $Z(\psi-\varphi)=0$ (c'est-à-dire que $\psi(0, \varepsilon)=\varphi(0, \varepsilon)$ ), et que $k$ est un réel positif non entier. L'opérateur linéaire $L$ défini par

$$
L(u)=-t \varphi u^{\prime}+k \psi u
$$

est bicontinu de $\mathcal{B}_{2}$ dans $\mathcal{B}_{1}$.

Démonstration. - Vérifions d'abord que $L$ est borné. On remarque que l'on a $\|t\|_{1}=r$, et on fait les majorations naturelles qui donnent :

$$
\|L(u)\|\left\|_{1} \leq\left(r\|\varphi\|_{1}+k\|\psi \psi\|_{1}\right) \cdot \frac{1}{2}\right\| u \|_{2} .
$$

On étudie ensuite l'inversibilité de $L$. À un élément $f$ de $\mathcal{B}_{1}$, cet inverse doit associer $u$, solution de l'équation $L(u)=f$. Il reste à vérifier que $u$ est bien défini de manière unique dans $\mathcal{B}_{2}$. La résolution explicite de l'équation $L(u)=f$ donne

où

$$
u=t^{k} \mathrm{e}^{G(t)} \int^{t} \frac{-\tau^{-k-1} \mathrm{e}^{-G(\tau)}}{\varphi(\tau)} f(\tau) \mathrm{d} \tau
$$

$$
G(t)=\int_{0}^{t} g(\tau) \mathrm{d} \tau \quad \text { et } \quad g=\frac{k S(\psi-\varphi)}{\varphi} .
$$

De plus, on exige que $u$ soit analytique en $t=0$, ce qui va déterminer la constante d'intégration. L'opérateur $L^{-1}$ sera donc obtenu comme composé de trois opérateurs : la multiplication par $\mathrm{e}^{-G} / \varphi$, l'opérateur $M$ défini ci-dessous et enfin la multiplication $\operatorname{par}^{G}$ :

$$
M(f)=t^{k} \int^{t}-\tau^{-k-1} f(\tau) \mathrm{d} \tau .
$$

Les lemmes 5.6, 5.2 et 5.1 montrent que $\mathrm{e}^{-G} / \varphi$ appartient à $\mathcal{B}_{1}$ et que le premier opérateur de multiplication est borné de $\mathcal{B}_{1}$ dans $\mathcal{B}_{1}$. De même, e ${ }^{G}$ appartient

TOME $129-2001-\mathrm{N}^{\mathrm{O}} 1$ 
à $\mathcal{B}_{2}$ et la multiplication par $\mathrm{e}^{G}$ est un opérateur borné de $\mathcal{B}_{2}$ dans $\mathcal{B}_{2}$. Reste à étudier l'opérateur $M$.

L'idée principale consiste à décomposer $f$ avec la formule de Taylor (voir lemme 5.8) à l'ordre $\bar{k}$, où $\bar{k}$ est l'entier immédiatement supérieur à $k$ :

$$
f=\sum_{i=0}^{\bar{k}} t^{i} Z S^{i} f+t^{\bar{k}+1} S^{\bar{k}+1} f .
$$

Un calcul immédiat donne pour tout $i$ entier

$$
M\left(t^{i}\right)=\frac{t^{i}}{k-i} .
$$

Soit $h$ un élément de $\mathcal{B}_{1}$. On s'intéresse à $M\left(t^{\bar{k}+1} h\right)$ : grâce à la positivité de $\bar{k}-k$, on a

$$
M\left(t^{\bar{k}+1} h\right)=t^{k} \int_{0}^{t} \tau^{\bar{k}-k} h(\tau) \mathrm{d} \tau .
$$

Soit $M_{n}$ le coefficient de $\varepsilon^{n}$ dans la série formelle $M\left(t^{\bar{k}+1} h\right)$, et $h_{n}$ celui de $h$. On calcule la norme de Nagumo en majorant la fonction bornée située sous l'intégrale :

$$
\begin{aligned}
\left\|M_{n}\right\|_{n / 2} & \leq \sup _{0 \leq|t|<r}|t|^{k} d(t)^{n / 2} \int_{0}^{t}|\tau|^{\bar{k}-k} \cdot\left|h_{n}(\tau)\right| \mathrm{d} \tau \\
& \leq \sup _{0 \leq|t|<r} r^{k} \int_{0}^{t} r^{\bar{k}-k}\left\|h_{n}\right\|_{n / 2} \frac{d(t)^{n / 2}}{d(\tau)^{n / 2}} \mathrm{~d} \tau \leq r^{\bar{k}+1}\left\|h_{n}\right\|_{n / 2} .
\end{aligned}
$$

En reprenant la décomposition de $f$ ci-dessus, ainsi que les lemmes 5.6, 5.1 et 5.7 , on obtient :

$$
\|M(f)\|_{1} \leq\left(\sum_{i=0, \ldots, \bar{k}} \frac{1}{|k-i|}\left(\frac{2 r}{\rho}\right)^{i}+\left(\frac{2 r}{\rho}\right)^{\bar{k}+1}\right) \cdot\|f\|_{1} .
$$

L'opérateur $M$ est donc borné de $\mathcal{B}_{1}$ dans $\mathcal{B}_{1}$. Mais, on remarque encore que $M(f)$ vérifie l'égalité différentielle

$$
-t M(f)^{\prime}+k M(f)=f .
$$

On en déduit donc que $M(f)^{\prime}=S(k M(f)-f)$, ce qui donne une majoration de $\left\|M(f)^{\prime}\right\|_{1}$. On obtient donc ainsi la majoration de l'opérateur $M$ de $\mathcal{B}_{1}$ dans $\mathcal{B}_{2}$.

\section{Existence de canards}

Pour montrer que l'existence de solutions formelles Gevrey-2 implique l'existence de vraies solutions, la méthode employée est semblable à celle évoquée par M. Canalis-Durand, J.-P. Ramis, R. Schäfke et Y. Sibuya dans [7]. Elle repose 
sur la théorie des fonctions Gevrey de B. Malgrange, J.-P. Ramis et Y. Sibuya (voir [12]).

On va démontrer l'existence des canards non pas pour le système (1) mais pour l'équation (9), pour conserver les notations précédentes. On rappelle que $r$ est un nombre réel strictement positif strictement inférieur au rayon de convergence de la fonction $z_{0}$ trouvée au paragraphe 4 .

8.1. Transformée de Borel-Laplace. - On choisit $\tilde{r}$ et $\beta$ tels que

$$
\beta<\delta \sqrt{d(\tilde{r})}
$$

On note $\gamma$ la constante $\delta \sqrt{d(\tilde{r})}$.

LEMme 8.1 (transformée de Borel). - Soit $\hat{z}=\sum z_{n}(t) \varepsilon^{n}$ un élément de $\mathcal{B}_{1}$. La série

$$
\sum_{n=1}^{\infty} \frac{z_{n}(t)}{\delta^{n}} \cdot \frac{\eta^{n-1}}{\frac{1}{2} \Gamma\left(\frac{1}{2} n\right)}
$$

converge pour tout $(t, \eta)$ dans un voisinage du pavé $|t| \leq \tilde{r},|\eta| \leq \beta$.

Démonstration. - Elle s'obtient par un calcul direct utilisant la majoration

$$
\sup _{|t| \leq \tilde{r}}\left|z_{n}(t)\right| \leq\left\|z_{n}\right\|_{n / 2}(d(\tilde{r}))^{-n / 2} \leq \frac{\|\hat{z}\|_{1}}{6}(d(\tilde{r}))^{-n / 2} \Gamma\left(\frac{n}{2}+1\right)
$$

Soient $\theta<\frac{1}{4} \pi$ et $\varepsilon_{0}>0$ fixés. On note $S$ le secteur

$$
S=\left\{\varepsilon \in \mathbb{C}, 0<|\varepsilon|<\varepsilon_{0},-\theta<\arg (\varepsilon)<\theta\right\} .
$$

DÉFInItion 8.2. - Soit $z$ une fonction analytique pour $|t| \leq \tilde{r}, \varepsilon \in S$. On dit qu'elle satisfait les majorations Gevrey-2 s'il existe des fonctions $z_{n}$, analytiques pour $|t| \leq \tilde{r}$, et des constantes $A$ et $\alpha$ telles que pour tout $t$ tout $\varepsilon$ et tout $N$, on ait

$$
\left|z(t, \varepsilon)-\sum_{n=0}^{N-1} z_{n}(t) \varepsilon^{n}\right| \leq A \alpha^{N} \Gamma\left(\frac{n}{2}\right) \varepsilon^{N} .
$$

La borne inférieure des nombres $\alpha$ s'appelle le type Gevrey de la fonction.

LEMme 8.3 (transformée de Laplace tronquée). — Soit $\hat{z}=\sum z_{n}(t) \varepsilon^{n}$ un élément de $\mathcal{B}_{1}$. La fonction

$$
\tilde{z}(t, \varepsilon)=z_{0}(t)+\int_{0}^{\beta} \sum_{n=1}^{\infty} \frac{z_{n}(t)}{\delta^{n}} \cdot \frac{\eta^{n-1}}{\frac{1}{2} \Gamma\left(\frac{n}{2}\right)} \exp \left(\frac{-\eta^{2}}{\varepsilon^{2}}\right) \mathrm{d} \eta
$$

- est analytique pour $|t| \leq \tilde{r}, \varepsilon \in S$;

- admet $\sum z_{n}(t) / \delta^{n} \cdot \varepsilon^{n}$ comme développement asymptotique;

- satisfait les majorations Gevrey.

TOME $129-2001-\mathrm{N}^{\mathrm{O}} 1$ 
Démonstration. - Les propriétés d'analyticité résultent du lemme précédent. Avant de démontrer les majorations Gevrey, il faut rappeler la formule

$$
\forall \varepsilon \in S, \quad \forall n>0, \quad \int_{0}^{\infty} \eta^{n-1} \exp \left(\frac{-\eta^{2}}{\varepsilon^{2}}\right) \mathrm{d} \eta=\frac{1}{2} \Gamma\left(\frac{n}{2}\right) \varepsilon^{n} .
$$

Celle-ci se démontre en prenant $v=\eta^{2} / \varepsilon^{2}$ pour nouvelle variable. Il faut aussi se rappeler que $\varepsilon^{2}$ a une partie réelle strictement positive et donc que le chemin d'intégration en $v$ est convenable.

Pour majorer le reste

$$
R_{N}(t, \varepsilon)=\tilde{z}(t, \varepsilon)-\sum_{n=0}^{N-1}\left(\frac{z_{n}(t)}{\delta^{n}}\right) \varepsilon^{n},
$$

on remplace $\varepsilon^{n}$ par l'intégrale donnée par la formule (14), coupée en $\int_{0}^{\beta}+\int_{\beta}^{\infty}$. Dans l'expression de $\tilde{z}$, on coupe ensuite la somme $\sum_{n=1}^{\infty}$ en deux morceaux $\sum_{n=1}^{N-1}$ et $\sum_{n=N}^{\infty}$. Ceci fait, les termes principaux se simplifient et le reste devient

$$
R_{N}(t, \varepsilon)=R_{N}^{1}(t, \varepsilon)+R_{N}^{2}(t, \varepsilon)
$$

avec

$$
\begin{aligned}
& R_{N}^{1}(t, \varepsilon)=-\sum_{n=1}^{N-1} \int_{\beta}^{\infty}\left(\frac{z_{n}(t)}{\delta^{n}}\right) \frac{\eta^{n-1}}{\frac{1}{2} \Gamma\left(\frac{n}{2}\right)} \exp \left(\frac{-\eta^{2}}{\varepsilon^{2}}\right) \mathrm{d} \eta \\
& R_{N}^{2}(t, \varepsilon)=\int_{0}^{\beta} \sum_{n=N}^{\infty}\left(\frac{z_{n}(t)}{\delta^{n}}\right) \frac{\eta^{n-1}}{\frac{1}{2} \Gamma\left(\frac{n}{2}\right)} \exp \left(\frac{-\eta^{2}}{\varepsilon^{2}}\right) \mathrm{d} \eta
\end{aligned}
$$

Dans chacun des termes, on peut majorer (pour des raisons différentes) $\eta^{n-1}$ par $\eta^{N-1} \beta^{n-N}$. La majoration (12) de $\left|z_{n}(t)\right|$ donne alors un majorant aussi bien de $\left|R_{N}^{1}(t, \varepsilon)\right|$ que de $\left|R_{N}^{2}(t, \varepsilon)\right|$ sous la forme

$$
\left|R_{N}^{i}(t, \varepsilon)\right| \leq\left(\sum_{n=1}^{\infty} \frac{\left\|\mid \hat{z}_{1}\right\|}{6} n\left(\frac{\beta}{\gamma}\right)^{n}\right) \frac{1}{\beta^{N}} \int_{0}^{\infty} \eta^{N-1}\left|\exp \left(\frac{-\eta^{2}}{\varepsilon^{2}}\right)\right| \mathrm{d} \eta,
$$

soit encore

$$
\left|R_{N}(t, \varepsilon)\right| \leq\left(\sum_{n=1}^{\infty} \frac{\left\|\mid \hat{z}_{1}\right\|}{6} n\left(\frac{\beta}{\gamma}\right)^{n}\right)\left(\frac{1}{\beta \sqrt{\cos (2 \theta)}}\right)^{N} \Gamma\left(\frac{N}{2}\right)|\varepsilon|^{N}
$$

qui est bien la majoration Gevrey recherchée.

REMARQUe 8.4. - Soit $S_{\alpha}$ le secteur

$$
S_{\alpha}=\left\{\varepsilon \in \mathbb{C}, 0<|\varepsilon|<\varepsilon_{0}, \alpha-\theta<\arg (\varepsilon)<\alpha+\theta\right\} .
$$

Si on définit $\tilde{z}_{\alpha}$ de la même façon que $\tilde{z}$, mais avec le segment $\left[0, \beta \mathrm{e}^{i \alpha}\right]$ comme chemin d'intégration, le lemme précédent reste valable pour la fonction $\tilde{z}_{\alpha}$, avec $\varepsilon \in S_{\alpha}$. Cependant, on peut comparer les différents $\tilde{z}_{\alpha}$ entre eux. 
LEMME 8.5. - Si $S_{\alpha_{1}} \cap S_{\alpha_{2}}$ est non vide, il existe des constantes $A$ et $\kappa$ telle que sur le domaine $|t| \leq \tilde{r}, \varepsilon \in S_{\alpha_{1}} \cap S_{\alpha_{2}}$, la différence $\left|\tilde{z}_{\alpha_{1}}-\tilde{z}_{\alpha_{2}}\right|$ est inférieure $\grave{a} A \exp \left(-\kappa^{2} / \varepsilon^{2}\right)$.

Démonstration. - Cette différence s'écrit comme l'intégrale, sur l'arc de cercle reliant $\beta \mathrm{e}^{i \alpha_{1}}$ et $\beta \mathrm{e}^{i \alpha_{2}}$, du produit de $\exp \left(-\eta^{2} / \varepsilon^{2}\right)$ par une fonction bornée. Pour $\varepsilon$ dans le domaine considéré, cette intégrale est exponentiellement petite.

\subsection{Quasi-solutions}

LEMME 8.6. - Soit $\hat{z}=\sum z_{n}(t) \varepsilon^{n}$ la série formelle, élément de $\mathcal{B}_{2}$ telle que $\sum\left(z_{n}(t) / \delta^{n}\right) \varepsilon^{n}$ soit une solution formelle de (9). La fonction $\tilde{z}$ définie par la formule (13) est alors une quasi-solution de l'équation (9) au sens suivant : si on pose

$$
\begin{aligned}
\tilde{w}(t, \varepsilon)=\frac{\varepsilon^{2}}{2} \tilde{z}^{\prime \prime}+\tilde{z} \tilde{z}^{\prime}+ & k t+(k+1) \tilde{z} \\
& -\Phi_{0}\left(t, \varepsilon, \tilde{z}, \varepsilon^{2} \tilde{z}^{\prime}\right)-\tilde{z}^{\prime} \Phi_{1}\left(t, \varepsilon, \tilde{z}, \varepsilon^{2} \tilde{z}^{\prime}\right),
\end{aligned}
$$

alors il existe des constantes $A$ et $\kappa$ telles que

$$
\forall t,|t|<\tilde{r}, \forall \varepsilon \in S, \quad|\tilde{w}(t, \varepsilon)| \leq A \exp \left(\frac{-\kappa^{2}}{\varepsilon^{2}}\right) .
$$

Démonstration. - Elle repose sur les résultats de B. Malgrange, J.-P. Ramis et Y. Sibuya énoncés dans [12], sur les fonctions Gevrey sur des secteurs autour de l'origine.

Soit $S_{\alpha_{i}}$ un bon recouvrement d'un voisinage de zéro par des secteurs (c'est le cas pour une sous-famille finie bien choisie de nos $S_{\alpha}$.) Si les $\tilde{w}_{\alpha_{i}}$ forment une famille de fonctions analytiques sur $S_{\alpha_{i}}$, ayant un développement asymptotique (au sens de Poincaré) à l'origine et telles que les différences $\tilde{w}_{\alpha_{i}}-\tilde{w}_{\alpha_{i+1}}$ soient exponentiellement petites, alors elles satisfont les majorations Gevrey à l'origine.

Nous définissons ici les $\tilde{w}_{\alpha}$ avec la formule (15), en remplaçant $\tilde{z}$ par $\tilde{z}_{\alpha}$.

On sait que les $\tilde{z}_{\alpha}$ ont un développement asymptotique (au sens de Poincaré) qui satisfait l'équation différentielle (9). On en déduit alors que $\tilde{w}_{\alpha}$ a un développement asymptotique nul.

D'autre part, on sait que $\hat{z}$ est un élément de $\mathcal{B}_{2}$. Donc $\hat{z}$ est élément de $\mathcal{B}_{1}$, ainsi que $\hat{z}^{\prime}$ et $\varepsilon^{2} \hat{z}^{\prime \prime}$ (voir le lemme 5.4). Le lemme précédent montre donc que $\tilde{z}_{\alpha_{i}}-\tilde{z}_{\alpha_{i+1}}, \tilde{z}_{\alpha_{i}}^{\prime}-\tilde{z}_{\alpha_{i+1}}^{\prime}$ et $\varepsilon^{2} \tilde{z}_{\alpha_{i}}^{\prime \prime}-\varepsilon^{2} \tilde{z}_{\alpha_{i+1}}^{\prime \prime}$ sont exponentiellement petits. De plus, en dérivant sous le signe $\sum$ et sous le signe $\int$, on montre que la fonction $\tilde{z}^{\prime}$, construite par transformée de Borel-Laplace tronquée à partir de $\hat{z}^{\prime}$ est la dérivée $\tilde{z}^{\prime}$ de la fonction $\tilde{z}$.

TOME $129-2001-\mathrm{N}^{\mathrm{O}} 1$ 
On majore maintenant les différences $\left|\tilde{w}_{\alpha_{i}}-\tilde{w}_{\alpha_{i+1}}\right|$ par les opérations habituelles pour montrer qu'elles sont exponentiellement petites dans les domaines considérés.

Le théorème de B. Malgrange, J.-P. Ramis et Y. Sibuya donne alors la conclusion du lemme.

LEMME 8.7. - La solution $z(t, \varepsilon)$ de l'équation (9) ayant pour conditions initiales les valeurs de $\tilde{z}$ et $\tilde{z}^{\prime}$ en $(0, \varepsilon)$ est un canard de (9), c'est-à-dire qu'il existe $\tilde{\tilde{r}}$ strictement positif et un secteur $S$ tels que $z$ est analytique pour $|t|<\tilde{\tilde{r}}$ et $\varepsilon \in S$, et a un développement asymptotique quand $\varepsilon$ tend vers zéro.

Démonstration. - Le lemme de Gronwall permet de majorer $|z-\tilde{z}|$ par une expression du type

$$
|z-\tilde{z}| \leq A_{1} \exp \left(\frac{-\kappa^{2}}{\varepsilon^{2}}\right) \exp \left(\frac{K}{\varepsilon^{2}} t\right)
$$

où $\kappa$ est donné par le lemme 8.6 et $K$ est une constante indépendante de $\varepsilon$. La majoration ci-dessus reste valable tant que $z$ et $\tilde{z}$ restent dans un domaine borné uniformément en $\varepsilon$. Il faut donc choisir $\tilde{\tilde{r}}<\tilde{r}$ de sorte que $-\kappa^{2}+K t$ soit toujours négatif.

Conclusion : après retour aux inconnues initiales $x, y$ et $z$, le lemme 8.7 donne le théorème 1.5 .

\section{Propriétés des canards : conjectures}

9.1. Caractère macrolocal du résultat. - Le théorème 1.5 est « macrolocal » au sens suivant : il annonce l'existence d'un voisinage (d'où le qualificatif «local») en $t$, indépendant de $\varepsilon$ (d'où le qualificatif « macro »). Ce résultat n'est pas entièrement satisfaisant quand on le confronte au problème posé initialement. En effet, si on se donne un candidat-canard (solution du système réduit), il est impossible avec ce théorème de savoir s'il est limite d'une famille de trajectoires du système initial quand $\varepsilon$ tend vers zéro.

Dans le cas où la dimension lente est 1 , le problème macrolocal est résolu avec beaucoup de généralité dans [7]. Le problème global est lui aussi résolu, au moins dans le cas d'un point tournant, dans [6]. Des compléments à cette étude globale peuvent aussi se trouver dans un article non encore publié de A. Fruchard et R. Schäfke, ou dans [9].

Conjecture 9.1. - Le domaine maximal (en $x, y, z)$ d'existence de canards se voit sur le système réduit, c'est-à-dire que tout candidat canard analytique est limite quand $\varepsilon$ tend vers zéro d'une solution du système initial, au moins dans le cas non résonnant. 
Cependant, les méthodes employées dans cet article sont inutilisables pour faire une démonstration globale. Déjà, dans la réduction du système (1) à une équation d'ordre deux (9), on utilise le théorème des fonctions implicites ; à cet endroit, il pourrait encore se faire que des méthodes géométriques puissent remplacer ces transformations, et permette de conserver le candidat-canard global. Mais la méthode de dilatation elle-même est une utilisation du théorème des fonctions implicites qui enlève tout espoir de démontrer ainsi des propriétés globales; on pourrait tout au plus donner une minoration explicite de la taille des canards.

9.2. Régularité des canards. — La démonstration du théorème (1.5) peut être améliorée de sorte qu'on montre que les solutions canards $z(t)$ sont de classe $S^{\infty}$, c'est-à-dire que les dérivées de $z$ ont des développements asymptotiques qui sont les dérivées des développements asymptotiques.

Ainsi, la méthode ne chasse que les canards $S^{\infty}$. Dans [5], un cas très particulier de l'étude précédente est étudié : c'est le cas où les fonctions $\Phi_{0}$ et $\Phi_{1}$ de l'équation (9) sont identiquement nulles. Dans cette situation, l'existence des canards $S^{\infty}$ ne pose aucun problème, car les solutions formelles sont très simples : elles sont polynomiales en $\varepsilon$ (et en $t$ ).

Cependant, tout le travail qui est fait ensuite dans [5] concernant l'enlacement des canards et l'existence de canards non $S^{1}$ reste valable : en prenant comme canard de référence le canard maximal donné par la conjecture 9.1 , on pourrait mener les mêmes calculs pour obtenir les résultats suivants :

Proposition 9.2. - Tout canard $S^{1}$ (c'est-à-dire dont les dérivées par rapport àt sont bornées uniformément en $\varepsilon$ ) a pour ombre (c'est-à-dire pour limite) l'une des deux trajectoires analytiques du système réduit.

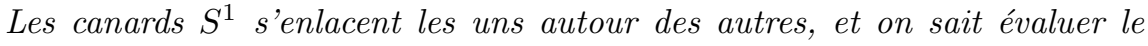
nombre de tours avec le paramètre $k$.

Dès que $k$ est supérieur à 3 , il existe des canards non $S^{1}$.

Le cas résonnant est, avec les méthodes ci-dessus, inaccessible (du moins semble-t-il). En effet, le système réduit n'a généralement pas de solution analytique tangente au sous-espace propre associé à la valeur propre $\lambda$. On ne dispose donc pas de la première approximation pour écrire des développements formels à coefficients analytiques. Il semble cependant que la bifurcation qui se produit quand le rapport des valeurs propres traverse une valeur entière soit intéressante. Le nombre d'enlacement des canards se modifie, et les raisonnements géométriques du type de ceux de [5] donneront peut-être des résultats.

TOME $129-2001-\mathrm{N}^{\mathrm{O}} 1$ 


\section{BIBLIOGRAPHIE}

[1] Arnold (V.I.) - Chapitres supplémentaires de la théorie des équations différentielles ordinaires, Éditions MIR, Moscou, 1980.

[2] Benoît (E.), Callot (J.-L.), Diener (F.), Diener (M.) - Chasse au canard, Collectanea Mathematica, t. 31-32 (1981), p. 37-119.

[3] Benô̂T (E.) - Systèmes lents-rapides dans $\mathbb{R}^{3}$ et leurs canards, in Troisième rencontre du Schnepfenried, p. 159-191, Astérisque, vol. 109-110, Soc. Math. France, 1983.

[4] Benoît (E.) - Canards de $\mathbb{R}^{3}$, Thèse d'état, Université de Nice, 1984.

[5] Benoît (E.) - Canards et enlacements, Publications IHES, t. 72 (1990), p. 63-91.

[6] Benoît (E.), Fruchard (A.), Schaefke (R.), Wallet (G.) - Solutions surstables des équations différentielles lentes-rapides à point tournant, Annales de la Faculté des Sciences de Toulouse, t. VII (4) (1998), p. $627-658$.

[7] Canalis-Durand (M.), Ramis (J.-P.), Schäfke (R.), Sibuya (Y.) - Gevrey solutions of singularly perturbed differential equations, J. reine angew. Math., t. 518 (2000), p. 95-129.

[8] Jones (C.K.R.T.) - Geometric singular perturbation theory, Lecture Notes in Math., vol. 1609, Springer, 1995.

[9] LIN (C.H.) - The sufficiency of Matkowsky condition in the problem of resonance, Trans. Amer. Math. Soc., t. 278 (2) (1983), p. 647-670.

[10] Lobry (C.), Sari (T.), Tounami (S.) - On Tykhonov's theorem for convergence of solutions of slow and fast systems, Electronic J. Diff. Equ., t. 19 (1998), p. 1-22.

[11] Malgrange (B.) - Sur le théorème de Maillet, Asymptotic Analysis, t. 2 (1989), p. 1-4.

[12] Malgrange (B.), Ramis (J.-P.) - Fonctions multisommables, Ann. Institut Fourier, Grenoble, t. 42 (1-2) (1992), p. 353-368.

[13] TAKens (F.) - Constrained equations; A study of implicit differential equations and their discontinuous solutions, in Structural Stability, the theory of catastrophes and applications in the sciences, Lecture Notes in Math., vol. 525, Springer Verlag, 1976.

[14] Wasow (W.) - Linear Turning Point Theory, Interscience, New York, 1985.

[15] Wechselberger (M.) - Singularly perturbed folds and canards in $\mathbb{R}^{3}$, Ph. D. thesis, Universität Wien, 1998. 